\title{
Treatment of Recurrent Tracheocutaneous Fistulas in the Irradiated Neck with a Two Layers-Two Flaps Combined Technique
}

\author{
M. Pignatti” ${ }^{1, \odot}$ G. Sapino ${ }^{2}$ M. Alicandri-Ciufelli3 $\quad$ F. Canzano ${ }^{3} \quad$ L. Presutti ${ }^{3} \quad$ G. De Santis ${ }^{2}$ \\ ${ }^{1}$ Department of Plastic Surgery, Policlinico di Sant'Orsola, DIMES, \\ University of Bologna, Bologna, Italy \\ 2Department of Plastic Surgery, Policlinico di Modena, \\ University of Modena and Reggio Emilia, Modena, Italy

\begin{abstract}
Address for correspondence Pignatti Marco, MD, FEBOPRAS, Chirurgia Plastica, Policlinico di Sant'Orsola, DIMES, University of Bologna, Via Giuseppe Massarenti, 9, Bologna 40138, Italy
\end{abstract} \\ (e-mail: mrpignatti@gmail.com).
}

${ }^{3}$ Department of Otolaryngology, Policlinico di Modena,

University of Modena and Reggio Emilia, Modena, Italy

Indian J Plast Surg:2020;53:423-426

\begin{abstract}
Keywords

- pedicle flap

- tracheocutaneous

fistula

- tracheostomy

- wound healing

The development of a tracheocutaneous fistula (TCF) is a well-documented complication after tracheostomy, especially in chronic morbid patients, in whom tubes or cannulas are left in place over time, or in irradiated patients.

Surgical treatments are therefore needed which range from simple curettage and dressings to local skin flaps, muscle flaps and, in the more complex cases, microsurgical free tissue transfers.

We present a novel combined technique used to successfully treat recurrent TCFs in irradiated patients, involving a superiorly based turnover fistula flap and a sternocleidomastoid transposition flap.
\end{abstract}

\section{Introduction}

Tracheostomy is usually temporary, and the removal of the cannula within days or weeks leads to spontaneous healing of the surgical wound.

In chronic morbid patients, in whom tracheostomy tubes are left in place over time, ${ }^{1}$ or in patients undergoing radiation therapies, the development of a tracheocutaneous fistula (TCF) is a well-documented complication. The incidence of chronic fistula can be as high as $29 \%$ and becomes even more common in the pediatric population. ${ }^{1,2}$ Phonation issues, mucopurulent secretions, skin irritation, aspiration, and pneumonia are possible consequences of $\mathrm{TCF}^{3}$

Surgical treatments are therefore needed and range from simple curettage and dressings to local skin flaps, muscle flaps and, in the more complex cases, microsurgical free tissue transfers. ${ }^{4}$ Especially in irradiated patients, due to the decreased wound healing potential, there is a high-rate of TCF recurrence. Surgical repair should therefore be multilayered and include well-vascularized tissue from the proximity. ${ }^{5}$
We present a novel combined technique, which is used to treat recurrent tracheocutaneous fistulas in irradiated patients, involving a superiorly based turnover fistula flap and a sternocleidomastoid transposition flap.

\section{Surgical Technique}

Under general anesthesia, an elliptical incision centered on the fistula is performed. The fistula is dissected from the surrounding tissues down to the anterior wall of the trachea. The fistula is subsequently split transversally, creating a caudal half that is excised and a cranial part that is turned down over the tracheal defect, as a superiorly based turnover flap, and sutured with PDS 4-0.

To provide additional coverage to the described tracheaplasty, the anterior border of the left sternocleidomastoid muscle is identified at the lateral border of the skin incision. The muscle is then released from its sternal attachment, harvested in a cranial direction while preserving its superior pedicle (from the occipital artery) and medial pedicle (from the superior thyroid artery), transposed over the repaired published online

July 28,2020
DOI https://doi.org/

$10.1055 / \mathrm{s}-0040-1714769$

ISSN 0970-0358. (c) 2020. Association of Plastic Surgeons of India.

This is an open access article published by Thieme under the terms of the Creative Commons Attribution-NonDerivative-NonCommercial-License, permitting copying and reproduction so long as the original work is given appropriate credit. Contents may not be used for commercial purposes, or adapted, remixed, transformed or built upon. (https://creativecommons.org/licenses/by-nc-nd/4.0/).

Thieme Medical and Scientific Publishers Pvt. Ltd. A-12, 2nd Floor, Sector 2, Noida-201301 UP, India 


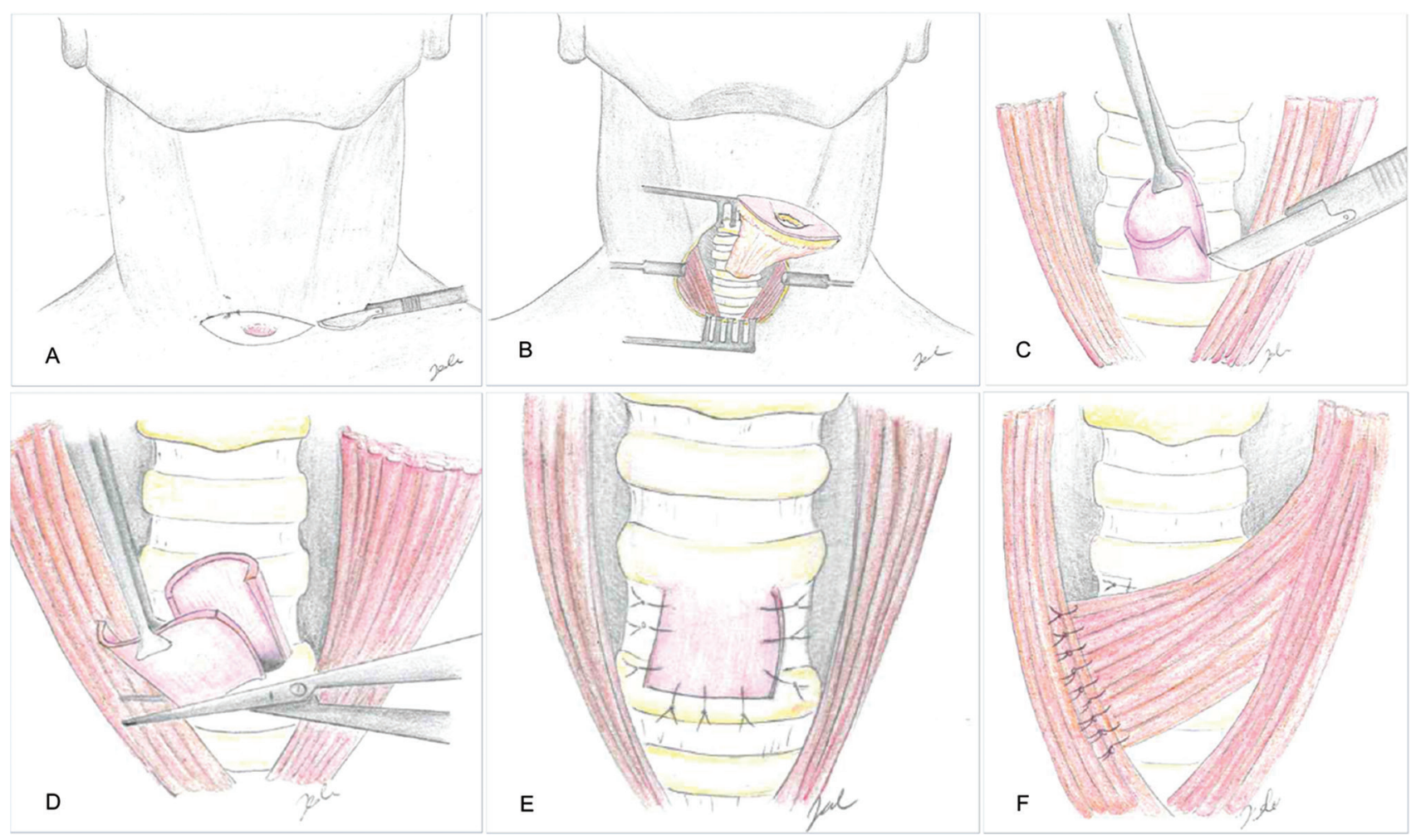

Fig. 1 (A) elliptical incision centered on the fistula. (B) The fistula is dissected from the surrounding tissues, down to the anterior wall of the trachea. (C) The fistula is split transversally. (D) The caudal half of the fistula is excised. (E) The cranial part is turned down over the tracheal defect as a superiorly based turnover flap and sutured. (F) The sternocleidomastoid muscle is transposed over the repaired tracheal defect and sutured.

tracheal defect, and sutured with Vicryl 4-0 to the available surrounding tissues. A small suction drain is positioned and kept until the output was less than $25 \mathrm{~mL} /$ day (-Fig. 1).

\section{Case 1}

A 64-year-old man, who was a heavy smoker, came to our attention, referring to the persistence of a $0.5 \mathrm{~cm}$ diameter TCF. Ten years before, he underwent subtotal laryngectomy (tracheohyoidoepiglottopexy) and right neck dissection, followed by radiotherapy for a squamocellular carcinoma. Since the first decannulation, he developed TCF and multiple unsuccessful treatment procedures, either with curettage and secondary intention healing or direct closure, were attempted over time. A superiorly based turnover fistula flap combined with a sternocleidomastoid transposition flap were used to cover the defect. Operating time was approximately 60 minutes. The patient was discharged on the first postoperative day. At 20 months follow-up, no recurrence of TCF was reported, with a good functional and aesthetic result (-Fig. 2).

\section{Case 2}

The same technique was used to treat a recurrent TCF of $1 \mathrm{~cm}$ in a 69-year-old male patient. He was operated several years before for a tracheal stenosis and the positioning of a Montgomery tracheal tube, which was kept for almost
5 years. The surgery was performed in 45 minutes and the patient was hospitalized for 2 days. Complete healing was achieved without complications. No recurrence occurred, with a follow-up of 14 months.

\section{Discussion}

The persistence of TCF is related to duration of cannulation, age at tracheostomy, and technique employed. ${ }^{6}$ When cannula has been present for a time longer than 16 weeks, the incidence of TCF can reach 70\%. ${ }^{7}$

Several local flaps have been described for the treatment of chronic TCF. Among them are rhomboid flap and Z-plasty, ${ }^{5}$ local turnover flap combined with pregrafted palatal mucosa, ${ }^{8}$ superiorly based turnover skin flap, ${ }^{1}$ and hinged skin flap. ${ }^{9}$

As authors agreed, reconstruction should be multilayered and the infrahyoid muscles around the trachea are usually approximated and sutured. In some cases, however, because of radiotherapy or previous surgery, those muscle fibers are damaged and not useful. Healthy, vascularized tissue needs to be brought from elsewhere.

The multilayered technique we describe here possesses some advantages.

The first incision is positioned horizontally and follows the lines used in previous surgeries in most patients (e.g., tracheotomy and anterior neck flaps for laryngectomies). This orientation, with the removal of the damaged, scarred area of 

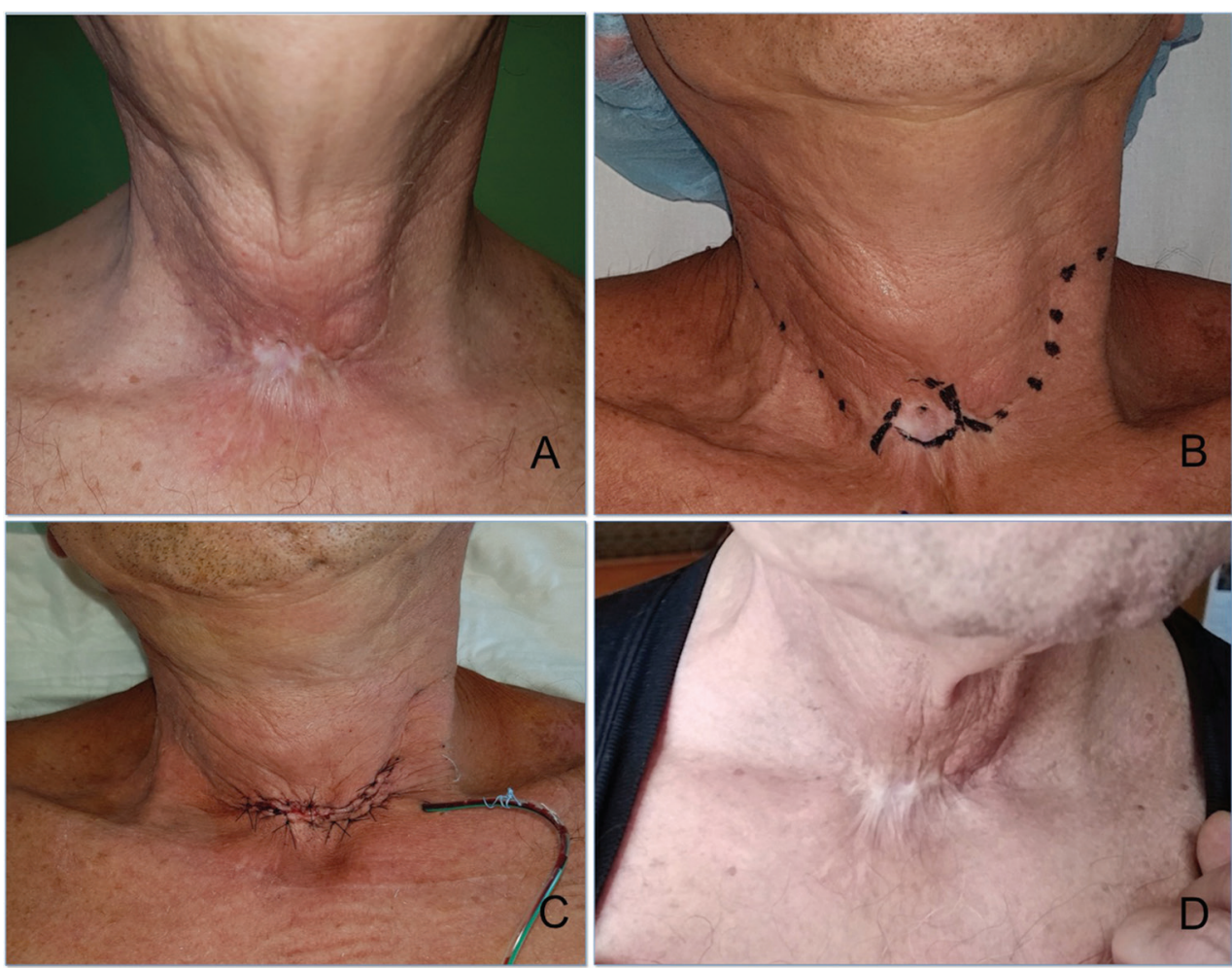

Fig. 2 (A) recurrent tracheocutaneous fistula after subtotal laryngectomy, radiotherapy and right neck dissection, and multiple closure attempts. (B) Preoperative markings: planned scarred skin excision around the fistula. Marked dots over previous neck surgical scars. (C) Immediate postoperative result. (D) Result at 20 months follow-up: excellent functional result without complications and a satisfied patient

the skin, also provides easy access to the trachea and the sternal and clavicular insertions of sternocleidomastoid muscles.

The TCF is due to the squamous epithelialization of the tracheostomy stoma tract. The use of a superiorly based flap made of the fistula tissue allows the repair of the tracheal mucosal defect with a similar quality tissue, grown from the mucosal epithelium with time, following the principles of "like with like" reconstruction.

This creates the basis for a first intention healing, preferable in patients with chronic TCF where previous attempts with curettage and secondary intention healing have been unsuccessful.

Once the prerequisites for a fast and safe healing of the trachea are set, in patients with other risk factors for TCF recurrence, a further prudential procedure is added.

The sternocleidomastoid muscle flap represents a wellknown option for head and neck defects but the donor site morbidity may include reduced head motion and aesthetic sequelae, and some authors therefore suggest to leave it as a back-up option. ${ }^{10}$ However, in selected cases (e.g., multioperated and irradiated patients), the advantages of this flap overcome the risks. The fibers of the muscle can provide adequate perfusion of the repaired tracheal tissues and at the same time mechanically reduce the risk of air leakage (cause of subcutaneous emphysema, pneumothorax or pneumomediastinum) by covering and somehow sealing (with fibrin and then scarring) the airway repair.

This flap is easy to access and harvest and may have a wide arc of rotation for different reconstructive needs.
We think that the described technique should be considered among the multiple possible solutions for recurrent TCF, especially in highly morbid irradiated patient, in which more complex reconstructions are not advisable, in order to provide a stable and effective coverage of the defect with a short and simple operation.

\section{Conflict of Interest}

None declared.

\section{References}

1 Feehs KR, Maslan JT, Kirse DJ. Superiorly-based turnover skin flap: Pediatric tracheocutaneous fistula closure. Int J Pediatr Otorhinolaryngol 2018;107:21-24

2 Gallagher TQ Hartnick CJ. Tracheocutaneous fistula closure. Adv Otorhinolaryngol 2012;73:76-79

3 Rennekampff HO, Tenenhaus M. Turnover flap closure of recalcitrant tracheostomy fistula: a simplified approach. Plast Reconstr Surg 2007;119(2):551-555

4 Royer AK, Royer MC, Ting JY, Weisberger EC, Moore MG. The use of a prefabricated radial forearm free flap for closure of a large tracheocutaneous fistula: a case report and review of the literature. J Med Case Reports 2015;9:251

5 Hernot S, Wadhera R, Kaintura M, et al. Tracheocutaneous fistula closure: comparison of rhomboid flap repair with $\mathrm{Z}$ plasty repair in a case series of 40 patients. Aesthetic Plast Surg 2016;40(6):908-913

6 Tasca RA, Clarke RW. Tracheocutaneous fistula following paediatric tracheostomy-a 14-year experience at Alder Hey Children's Hospital. Int J Pediatr Otorhinolaryngol 2010;74(6): 711-712 
7 Kulber H, Passy V. Tracheostomy closure and scar revisions. Arch Otolaryngol 1972;96(1):22-26

8 Kitazawa T, Shiba M. Closure of a tracheocutaneous fistula with a local turnover flap combined with pregrafted palatal mucosa: a case report. Eplasty 2016;16:e30

9 Kamiyoshihara M, Nagashima T, Takeyoshi I. A novel technique for closing a tracheocutaneous fistula using a hinged skin flap. Surg Today 2011;41(8):1166-1168
10 Jones LF, Farrar EM, Roberts DJH, Moor JW. Revisiting the sternocleidomastoid flap as a reconstructive option in head and neck surgery. J Laryngol Otol 2019;(e-pub ahead of print). doi: $10.1017 /$ S0022215119001592 